\title{
Is it Possible to Predict Preeclampsia Early by Maternal Pregnancy Associated Plasma Protein-A and Uterine Artery Doppler? A Randomized-Controlled Trial \\ Abeer Elshabacy ${ }^{1}$, Hala A.E. Tabl ${ }^{2}$, Sahar H. Quashwa ${ }^{3}$ \\ Departments of ${ }^{1}$ Obstetrics \& Gynecology, ${ }^{2}$ Radiology and \\ ${ }^{3}$ Clinical Pathology, Benha Teaching Hospital, Egypt \\ *Corresponding author: Abeer Elshabacy, Mobile: (+20) 01223331182, E-Mail: abear.elshabacy@ gmail.com
}

\begin{abstract}
Background: Hypertensive disorders in pregnancy, along with IUGR and low birth weight, are among the major causes of maternal mortality and morbidity. It is one of the most common pregnancy complications. Its incidence ranges between $10-12 \%$ of all pregnancies. Early detection of preeclampsia (PE) is of utmost importance to start early intervention that prevents maternal and fetal comorbidities and decreases maternal and fetal mortality.

Objectives: To predict the role of maternal serum pregnancy-associated plasma protein-A (PAPP-A) and uterine artery Doppler in females between 11-14 weeks of gestation for early detection of preeclampsia.

Patients and methods: This randomized-controlled trial was done at Benha Teaching Hospital Department of Obstetrics \& Gynecology over 200 pregnant women with gestational ages of 11-14 weeks. Blood pressure was recorded, uterine artery Doppler was done, and a blood sample for PAPP-A was drawn.

Results: Uterine artery pulsatility index (PI) at 11-14 weeks of gestation is a good screening method for preeclampsia. The mean pulsatility index was more valid than PAPP-A. However, the combination of both in addition to patient history and blood pressure examination is more predictive.

Conclusion: Our study concluded that at 11-14 weeks of gestation, uterine artery Doppler and serum level of maternal PAPPA-A were good screening methods for early prediction of preeclampsia.
\end{abstract}

Keywords: Preeclampsia, PAPP-A, uterine artery Doppler.

\section{INTRODUCTION}

Preeclampsia (PE) is a pregnancy-explicit condition described by hypertension and proteinuria that disappear after delivery. PE is associated with high prevalence in a primigravida with increased morbidity and mortality risk to both the mother and the fetus, in addition to an increased incidence of preterm birth, perinatal death, and intrauterine growth restriction ${ }^{(\mathbf{1})}$. It is a multisystem maternal condition that only affects pregnant women, and it typically happens after 20 weeks of pregnancy. Preeclampsia is more common in developing countries than it is in developed countries $(1.8-16.7 \%)^{(2)}$.

The exact pathogenesis of preeclampsia remains unknown. In these cases, inadequate trophoblastic invasion is suspected. The placental abnormal development results in persistently high resistance to uteroplacental circulation blood flow ${ }^{(3)}$. Additionally, specific proteins in the circulation of the pregnant woman were found to be altered, as pregnancy associated plasma protein-A (PAPP-A), soluble fms-like tyrosine kinase-1 (sFlt-1), and placental growth factor (PlGF). Their alterations help in their use as biomarkers for preeclampsia prediction ${ }^{(4)}$.

Through the maternal spiral arteries, the fetus must obtain enough oxygen and nutrients in a normal pregnancy. The spiral arteries should be converted from high-resistant, low-capacity blood vessels to lowresistant, high-capacity blood vessels to ensure this. At 8-12 weeks of pregnancy, extra-villous trophoblastic cells must invade the decidual part of the spiral arteries to cause vascular remodeling ${ }^{(5)}$. A phase begins near the first-trimester end and lasts until the pregnancy is completed $(18-20 \text { weeks })^{\left({ }^{(}\right)}$. When preeclampsia complicates pregnancy, the process of vascular remodeling that occurs to the spiral arterioles becomes limited to the superficial part of the decidua, so the placenta becomes hypoxic and under-perfused. This can be detected by Doppler velocimetry of uterine arteries at the 11th week of gestation which will reveal high resistance in the maternal spiral arteries ${ }^{(7)}$. Usually, the flow in the uterine artery shows decreased impedance, which Doppler can measure; however, it increases in preeclampsia $^{(8)}$.

The trophoblastic cells produce pregnancyassociated plasma protein-A (PAPP-A), which is a 1628 amino acid peptide linked by a disulfide bond. It has an action on insulin-like growth factor binding proteins cleavage, so it regulates fetal growth ${ }^{(9)}$. In the first trimester, low maternal serum PAPP-A levels in chromosomally normal pregnancies have recently been linked to an increased risk of PE ${ }^{(\mathbf{1 0})}$. This fact can be explained by the insulin-like growth factor role in trophoblastic invasion ${ }^{(11)}$.

This article is an open access article distributed under the terms and conditions of the Creative Commons Attribution (CC BY-SA) license (http://creativecommons.org/licenses/by/4.0/) 
The objectives of this study were to predict the role of maternal serum pregnancy-associated plasma proteinA (PAPP-A) and uterine artery Doppler in females between 11-14 weeks of gestation for early detection of preeclampsia.

\section{PATIENTS AND METHODS}

This randomized-controlled trial was conducted in Obstetrics \& Gynecology Department Benha Teaching Hospital. It included two hundred women.

Inclusion criteria: Primigravida, single fetus pregnancy and gestational age between 11-14 weeks.

Exclusion criteria: Multiple pregnancies, multipara pregnant woman, gestational age $>16$ or $<14$ weeks, threatened abortion, recurrent miscarriage, any congenital abnormalities, Molar pregnancy, chromosomal abnormality, chronic hypertension and comorbid conditions (DM, renal, cardiovascular, and thyroid disorders).

\section{Ethical consent}

An approval of the study was obtained from Benha Teaching Hospital Academic and Ethical Committee. Every patient signed an informed written consent for acceptance of the operation.

All patients were subjected to the following:

- A full history taking including personal, menstrual, obstetric, past, present, and family history.

- General, abdominal (obstetric), and pelvic examinations, were done to exclude those not fitting with the abovelisted criteria
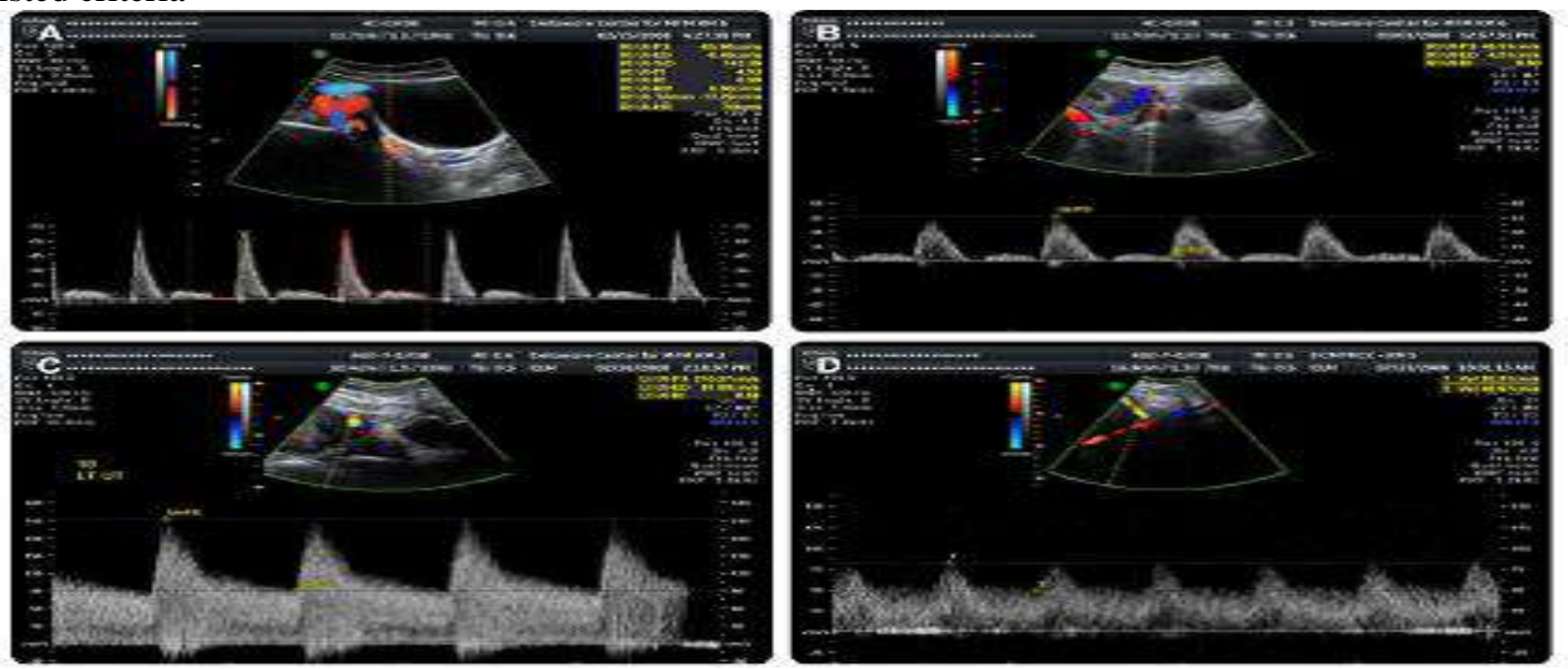

Figure (1): Uterine artery Doppler ultrasound in the nonpregnant and pregnant patient. (A) Nonpregnant patient. (B) First trimester. (C) Second trimester. (D) Third trimester ${ }^{(14)}$.
- Complete blood picture, fasting and 2-hour postprandial blood sugar, RH, urine analysis, and liver and kidney functions.

- Under complete aseptic conditions, a venous blood sample was obtained at 11-14 weeks gestation and allowed to clot in a non-heparinized tube and centrifuged. PAPP-A was measured on maternal serum by $\operatorname{ELISA}^{(\mathbf{1 2})}$.

- The PAPP-A ELISA Kit (IBL INTERNATIONAL) is a solid phase enzyme-linked immunosorbent assay (ELISA) based on sandwich principle.

- The microtiter wells were coated with a polyclonal anti PAPP-A antibody. An aliquot of patient sample was incubated in the coated well assay buffer. After incubation the unbound material was washed off. In the second incubation step a sandwich complex was formed with a polyclonal anti PAPP-A antibody peroxidase conjugate. The substrate solution was added, then the reaction was stopped by stop solution, the intensity of the color developed was proportional to the concentration of PAPP-A in the patient. The range of this assay is between 0.133 microgram / $\mathrm{ml} \mathrm{-30} \mathrm{microgram} / \mathrm{ml}^{(13)}$.

- Ultrasound examination to assess; gestational age, viability, nuchal translucency, and anomaly scan.

- Uterine artery Doppler was done to measure right and left uterine arteries pulsatility index (PI) and resistant index (RI.). All patients were followed up till term for the development of preeclampsia. 


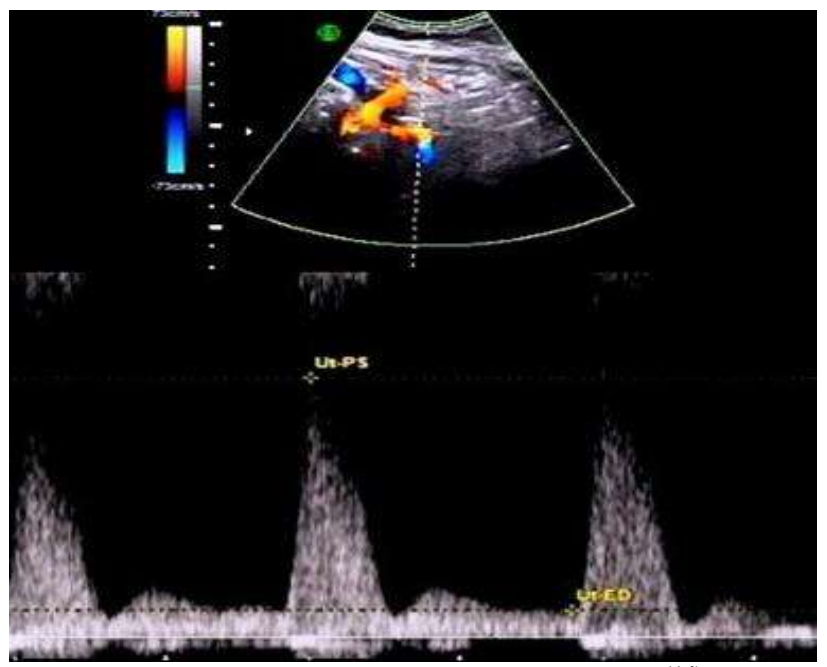

Figure (2): Uterine artery notching ${ }^{(14)}$.

\section{Statistical analysis}

The collected data were coded, processed and analyzed using the SPSS (Statistical Package for Social Sciences) version 22 for Windows ${ }^{\circledR}$ (IBM SPSS Inc, Chicago, IL, USA). Data were tested for normal distribution using the Shapiro Walk test. Qualitative data were represented as frequencies and relative percentages. Chi square test $(\chi 2)$ to calculate difference between two or more groups of qualitative variables. Quantitative data were expressed as mean \pm SD (Standard deviation).
Independent samples t-test was used to compare between two independent groups of normally distributed variables (parametric data). $\mathrm{P}$ value $\leq 0.05$ was considered significant.

\section{RESULTS}

Two hundred antenatal women 11-14 weeks of gestation were studied and subjected to screening of serum PAPP-A and uterine artery Doppler ultrasound. In this study, 14 out of 200 women developed preeclampsia.

Table (1): Comparison between normal \& pre-eclamptic cases regarding clinico-epidemiological data

\begin{tabular}{|c|c|c|c|c|}
\hline \multirow[t]{2}{*}{ Variable } & \multicolumn{2}{|c|}{ Mean \pm SD } & \multirow{2}{*}{$\begin{array}{l}\text { Student } \\
\text { t-test }\end{array}$} & \multirow[t]{2}{*}{$\mathbf{P}$} \\
\hline & $\begin{array}{c}\text { Normal } \\
(\text { no. }=186)\end{array}$ & $\begin{array}{c}\text { Preeclampsia } \\
\text { (no.=14) }\end{array}$ & & \\
\hline Maternal age (yrs.) & $29.6 \pm 5$ & $28.2 \pm 4$ & 0.72 & 0.47 \\
\hline Gestational age at recruitment (wks.) & $12.4 \pm 1.1$ & $12.2 \pm 1.3$ & 0.46 & 0.65 \\
\hline Gestational age at delivery (wks.) & $39 \pm 1.3$ & $36 \pm 0.9$ & 5.98 & $<0.001(\mathrm{HS})$ \\
\hline Weight (kg) & $67.8 \pm 9$ & $69.1 \pm 9$ & 0.37 & 0.71 \\
\hline Height (m) & $159 \pm 7$ & $157 \pm 5$ & 0.74 & 0.46 \\
\hline BMI $\left(\mathrm{kg} / \mathbf{m}^{2}\right)$ & $26.9 \pm 4$ & $28 \pm 3$ & 0.71 & 0.48 \\
\hline Birth weight (gm.) & $3100 \pm 160$ & $2800 \pm 200$ & 4.7 & $<0.001(\mathrm{HS})$ \\
\hline SBP (mmHg) & $118 \pm 4$ & $150 \pm 10$ & 17.76 & $<0.001(\mathrm{HS})$ \\
\hline DBP (mmHg) & $71.8 \pm 8$ & $99 \pm 7$ & 8.74 & $<0.001(\mathrm{HS})$ \\
\hline MABP (mmHg) & $87 \pm 10$ & $116 \pm 7$ & 7.52 & $<0.001(\mathrm{HS})$ \\
\hline PAPP-A $(\mu \mathrm{g} / \mathrm{ml})$ & $2.3 \pm 0.3$ & $1.1 \pm 0.2$ & 2.17 & $0.03(\mathrm{~s})$ \\
\hline PI of UA Doppler & $1.7 \pm 0.05$ & $2.97 \pm 0.2$ & 6.65 & $<0.001(\mathrm{HS})$ \\
\hline
\end{tabular}

In the present study, all women were Egyptian, primigravida, had a singleton pregnancy, none of the women enrolled were diabetic, had renal disease, or had more than 39 years. The mean age of the women in our study who developed preeclampsia was 28 years, while those who didn't develop preeclampsia were 29 years. The mean BMI of women with preeclampsia was $28 \mathrm{~kg} / \mathrm{m}^{2}$. On the other hand, those who didn't develop were $26.9 \mathrm{~kg} / \mathrm{m}^{2}$. In this study, the median serum PAPP-A was lower in cases that developed preeclampsia than non-pre-eclamptic women (1.1 and 2.3, respectively). The differences were statistically significant. Regarding the Doppler velocimetry in the current study, the mean pulsatility index (PI) of the uterine arteries was significantly higher in cases that developed preeclampsia than those who did not $(2.97 \pm 0.2$ and $1.7 \pm 0.5$, respectively) as shown in table (1). 
Table (2): Correlation of mean PAPP-A and mean PI with clinico- epidemiological data of pre-eclamptic case

\begin{tabular}{|l|c|c|c|c|}
\hline \multirow{2}{*}{ Variables (no.=14) } & \multicolumn{2}{c|}{ PAPP-A } & \multicolumn{2}{c|}{ Mein PI } \\
\cline { 2 - 4 } & $\mathbf{r}$ & $\mathbf{p}$ & $\mathbf{P}$ & $\mathbf{P}$ \\
\hline Maternal age (years) & 0.13 & $>0.05$ & 0.25 & $>\mathbf{0 . 0 5}$ \\
\hline Gestational age at recruitment (weeks) & 0.09 & $>0.05$ & 0.08 & $>\mathbf{0 . 0 5}$ \\
\hline Gestational age at delivery(weeks) & -0.13 & $>0.05$ & -0.07 & $>\mathbf{0 . 0 5}$ \\
\hline Weight $(\mathbf{~ k g})$ & 0.17 & $>0.05$ & -0.12 & $>\mathbf{0 . 0 5}$ \\
\hline Height $(\mathbf{c m})$ & 0.16 & $>0.05$ & -0.10 & $>\mathbf{0 . 0 5}$ \\
\hline BMI $\left(\mathbf{k g} / \mathbf{m}^{2}\right)$ & 0.18 & $>0.05$ & -0.13 & $>\mathbf{0 . 0 5}$ \\
\hline birth weight & 0.10 & $>0.05$ & 0.20 & $>\mathbf{0 . 0 5}$ \\
\hline SBP $(\mathbf{m m H g})$ & 0.79 & $<0.05(\mathrm{~S})$ & 0.12 & $>\mathbf{0 . 0 5}$ \\
\hline DBP $(\mathbf{m m H g})$ & 0.14 & $>0.05$ & 0.13 & $>\mathbf{0 . 0 5}$ \\
\hline MABP $(\mathbf{m m H g})$ & -0.16 & $>0.05$ & 0.18 & $>\mathbf{0 . 0 5}$ \\
\hline PAPP-A $(\boldsymbol{\mu g} / \mathbf{m l})$ & - & - & -0.14 & $>\mathbf{0 . 0 5}$ \\
\hline
\end{tabular}

Cases of preeclampsia, PAPP-A were significantly correlated positively with SBP by using the Spearman correlation test. No significant correlation regarding other variables (Table 2).

Table (3): The validity of mean PI \& PAPP-A in cases of preeclampsia

\begin{tabular}{|l|c|c|}
\hline \multicolumn{1}{|c|}{ Variables } & Mean- PI & PAPP-A \\
\hline Best cut off & 2.6 & $\mathbf{1 . 1}$ \\
\hline The area under the curve & 0.97 & $\mathbf{0 . 4 6}$ \\
\hline Sensitivity & $87 \%$ & $\mathbf{6 0 \%}$ \\
\hline Specificity & $96 \%$ & $\mathbf{5 3 \%}$ \\
\hline PPV & $88 \%$ & $\mathbf{5 7 \%} V$ \\
\hline NPV & $98 \%$ & $\mathbf{7 0 \%}$ \\
\hline Accuracy & $90 \%$ & $\mathbf{5 5 \%}$ \\
\hline
\end{tabular}

Table (3) showed that mean PI was more valid than PAPP-A in the prediction of preeclampsia.

\section{DISCUSSION}

Preeclampsia (PE) is a condition that involves many systems in the body. It was defined as hypertension that occurs after 20 weeks of pregnancy and is accompanied by proteinuria. PE definition has been broadened in recent years to include hypertension alone with pregnancy. PE affects around two to five percent of females during pregnancy. It is one of the major maternal and perinatal comorbidity and mortality causes, mainly when it occurs early in pregnancy ${ }^{(\mathbf{1 5})}$.

PE can be sub-divided into: Early (delivery $<34 \pm$ 0 weeks gestation), preterm (delivery $<37 \pm 0$ weeks gestation), late (delivery $\geq 34 \pm 0$ weeks gestation), and term (delivery $\geq 37 \pm 0$ weeks gestation). These subdivisions are not exclusive. Early-onset $\mathrm{PE}$ is accompanied by short- and long-term risks for both the mother and the fetus ${ }^{(16,17)}$.

Although the exact cause of preeclampsia is unknown, the most widely accepted theory indicates a process of two stages. The first is due to the shallow trophoblast invasion due to the spiral arteries insufficient remodeling. In the second stage, the maternal response to endothelial dysfunction is characterized by angiogenic and anti-angiogenic factors imbalance resulting in preeclampsia's clinical features ${ }^{(\mathbf{1 8}, \mathbf{1 9})}$.
Despite improvements in the understanding of PE pathophysiology, prediction and prevention remain elusive. The desire to diagnose women at a higher risk of developing PE enhances effective PE prediction in the first trimester of pregnancy, so specific interventions can be started early to improve placentation and minimize the disease incidence. Furthermore, recognizing patients who are "at-risk" would allow for more targeted prenatal screening and follow-up to diagnose and treat PE as early as possible ${ }^{(\mathbf{1 5})}$.

It is unlikely to find a single effective, accurate test useful clinically to predict early preeclampsia ${ }^{(20,21)}$. Many markers have been studied to screen PE in recent years, so combining many parameters in a model has become popular. Whether based on maternal demographics and background, that can alone detect, with a high false-positive rate, one-third of PE cases ${ }^{\text {(22). }}$ To enhance preeclampsia detection, many researchers have combined patient history with biophysical markers such as mean arterial blood pressure ${ }^{(23)}$, maternal cardiac output ${ }^{(24)}$, uterine artery Doppler ${ }^{(22)}$, brain hemodynamic measurements ${ }^{(25)}$, and more recently, pulse wave analysis (26). Furthermore, panels of molecular markers (circulating mRNA) have been studied and associated with PE development in addition to biochemical markers 
such as fetal and placental products, renal or endothelial damage markers, angiogenic and anti-angiogenic factors, and some oxidative stress ${ }^{(27,28)}$. These methods have been combined with maternal variables to derive a logistic regression-based model for calculating the detection rate (DR) of preeclampsia.

The present study is a randomized clinical trial conducted At Benha Teaching Hospital includes 200 women. These 200 cases were examined using transabdominal ultrasound and Doppler, a sample of maternal blood was collected (11wk-14wk gestation), and PAPPA was assayed by ELISA. We aimed to detect the accuracy of uterine artery Doppler measurement and serum PAPP-A between (11th and 14th week) of pregnancy for early prediction of preeclampsia.

Maternal age and BMI showed no significant differences between preeclampsia cases and normal females $(\mathrm{P}>0.05$.)

The mean SBP was $118 \pm 4 \mathrm{mmHg}$ in those who did not develop preeclampsia and $150 \pm 10 \mathrm{mmHg}$ in those who developed preeclampsia (p-value $<0.05$ ). DBP was $71.8 \pm 8 \mathrm{mmHg}$ in those who did not develop preeclampsia and $99 \pm 7 \mathrm{mmHg}$ in those who developed preeclampsia $(\mathrm{p}<0.05)$. These findings are similar to the results of Wright et al. ${ }^{(29)}$.

In this study, we found a statistical difference in the mean pulsatility index (PI) of the uterine arteries in cases that developed preeclampsia than non-preeclamptic cases $(2.97 \pm 0.2$ and $1.7 \pm 0.5$, respectively). These findings are similar to the results of Pedroso et al. ${ }^{(4)}$. On the other hand, Aksornphusitaphong et al. ${ }^{(30)}$ found that uterine artery PI and serum histidine-rich glycoprotein (HRG) combination is ineffective to predict preeclampsia at the first-trimester screening.

According to the current study, median serum PAPP-A was significantly lower in cases of preeclampsia than in non-preeclamptic cases $(1.5$ and 2.2, respectively). The findings of this study correlate with several previous studies, indicating that a low maternal serum PAPP-A level at 11-14 weeks of gestation is linked to an increased risk of PE later on ${ }^{(31,32)}$.

In cases of preeclampsia, PAPP-A was significantly correlated positively with SBP by using the Spearman correlation test. No significant correlation regarding other variables. Our results agree with Pedroso et al. (4) regarding Doppler's study of uterine artery pulsatility and notching and with that of Luewan $\boldsymbol{e t} \boldsymbol{a l}$. ${ }^{(31)}$ and Antsaklis et al. ${ }^{(32)}$ as regards PAPP-A.

It is essential to predict preeclampsia as early as possible to prevent or at least decrease its complications. It is also considered a challenge in clinical practice. Different screening methods have been discovered as maternal characteristics and history, which have poor sensitivity, uterine artery Doppler, and other markers. There is a direct link between PE and fetal growth restriction (FGR) development and elevated mean uterine artery PI in the first trimester. However, it has a low positive predictive value and sensitivity when used alone as a screening test. Combining uterine artery Doppler, maternal risk factors, and mean arterial pressure, with or without circulating biomarkers, improves sensitivity and minimizes false-positive results ${ }^{(27)}$, which is consistent with our findings.

\section{CONCLUSION}

Early detection of high-risk pregnant women for developing preeclampsia is essential to reduce the risk of its occurrence and complications for both the mother and the fetus. Screening at 11-14 weeks of pregnancy using uterine artery Doppler pulsatility index (PI) and PAPP-A serum level help detecting high-risk females, who may benefit from early aspirin use, and those who are at risk of premature delivery, which accounts for the majority of the disease morbidity and mortality. Besides, we found that the mean pulsatility index is more valid than PAPPA in screening for preeclampsia.

\section{REFERENCES}

1. Wang A, Rana S, Karumanchi S (2009): Preeclampsia: the role of angiogenic factors in its pathogenesis. Physiology, 24 (3): 147-158.

2. Piccoli G, Daidola G, Attini $R$ et al. (2013): Kidney biopsy in pregnancy: evidence for counselling? A systematic narrative review. BJOG: An International Journal of Obstetrics \& Gynaecology, 120 (4): 412-427.

3. Karumanchi $\mathbf{S}$ (2016): Angiogenic factors in preeclampsia: from diagnosis to therapy. Hypertension, 67 (6): 1072-1079.

4. Pedroso M, Palmer K, Hodges $R$ et al. (2018): Uterine artery Doppler in screening for preeclampsia and fetal growth restriction. Revista brasileira de ginecologia e obstetricia, 40 (5): 287-293.

5. Burton G, Jauniaux E (2018): Pathophysiology of placental-derived fetal growth restriction. American Journal of Obstetrics and Gynecology, 218 (2): 745-761.

6. Roberts V, Morgan T, Bednarek P et al. (2017): Early first trimester uteroplacental flow and the progressive disintegration of spiral artery plugs: new insights from contrastenhanced ultrasound and tissue histopathology. Human Reproduction, 32 (12): 2382-2393.

7. Brosens I, Puttemans P, Benagiano G (2019): Placental bed research: I. The placental bed: from spiral arteries remodeling to the great obstetrical syndromes. American Journal of Obstetrics and Gynecology, 221 (5): 437-456.

8. Albaiges G, Missfelder-Lobos H, Lees C et al. (2000): One-stage screening for pregnancy complications by color Doppler assessment of the uterine arteries at 23 weeks' gestation. Obstetrics \& Gynecology, 96 (4): 559-564.

9. Grill S, Rusterholz $\mathrm{C}$, Zanetti-Dällenbach $\mathrm{R}$ et al. (2009): Potential markers of preeclampsia-a review. Reproductive Biology and Endocrinology, 7 (1): 1-14. 


\section{https://ejhm.journals.ekb.eg/}

10. Ranta J, Raatikainen $K$, Romppanen J et al. (2011): Decreased PAPP-A is associated with preeclampsia, premature delivery and small for gestational age infants but not with placental abruption. European Journal of Obstetrics \& Gynecology and Reproductive Biology, 157 (1): 48-52.

11. Kumar M, Sharma K, Singh $\mathrm{R}$ et al. (2016): Role of maternal factors, PAPP-A, and Doppler in screening for early-and late-onset pregnancy hypertension in Asian population. Hypertension in Pregnancy, 35 (3): 382-393.

12. Johns J, Muttukrishna Johns, Lygnos M (2007): Maternal serum hormone concentrations for prediction of adverse outcome in threatened miscarriage. Reproductive Biomedicine Online, 15: 413-421.

13. Qiu-Ping Q, Christiansen M, Oxvig C et al. (1997): PAPP-A Ultrasensitive ELISA. Clinical Chemistry, 43 (12): 2323-2332.

14. Anwar N, Chowdhury T, Choudhury T (2020): Uterine Artery Doppler Screening in 2nd Trimester of Pregnancy for Prediction of Pre-eclampsia and Fetal Growth Restriction. Journal of Advances in Medicine and Medical Research, 32 (13): 7-22.

15. Poon L, Shennan A, Hyett J et al. (2019): The International Federation of Gynecology and Obstetrics (FIGO) initiative on pre-eclampsia: A pragmatic guide for first trimester screening and prevention. Int $\mathrm{J}$ Gynecol Obstet., 145: 1-33.

16. Lisonkova $S$, Joseph $K$ (2013): Incidence of preeclampsia: risk factors and outcomes associated with earlyversus lateonset disease. American Journal of Obstetrics and Gynecology, 209 (6): 544-e1.

17. Lisonkova S, Sabr Y, Mayer C et al. (2014): Maternal morbidity associated with early-onset and late-onset preeclampsia. Obstetrics \& Gynecology, 124 (4): 771-781.

18. Redman C, Sargent I (2005): Latest advances in understanding preeclampsia. Science, 308 (5728): 15921594.

19. Chaiworapongsa T, Chaemsaithong P, Yeo L (2014): Pre-eclampsia part 1: current understanding of its pathophysiology. Nature Reviews Nephrology, 10 (8): 466480 .

20. Dawonauth L, Jeeawoody R, Jankee S et al. (2019): High rate of early onset preeclampsia in Mauritius. Journal of Reproductive Immunology, 136: 102611-6.

21. Li M, Qian L, Yu J et al. (2020): Interleukin-35 inhibits human umbilical vein endothelial cell injury induced by sera from pre-eclampsia patients by up-regulating S100A8 protein expression. Hypertension in Pregnancy, 39 (2): 126138.
22. Sepúlveda Martínez A, Rencoret $G$, Silva $M$ et al. (2019): First trimester screening for preterm and term pre eclampsia by maternal characteristics and biophysical markers in a low risk population. J. Obstet Gynaecol Res., 45: 104-112.

23. Gasse C, Boutin A, Cote $M$ et al. (2018): First-trimester mean arterial blood pressure and the risk of preeclampsia: The Great Obstetrical Syndromes (GOS) study. Pregnancy Hypertension, 12: 178-182.

24. Tay J, Foo L, Masini G et al. (2018): Early and late preeclampsia are characterized by high cardiac output, but in the presence of fetal growth restriction, cardiac output is low: insights from a prospective study. American Journal of Obstetrics and Gynecology, 218 (5): 517-e1.

25. Riskin-Mashiah S, Belfort M, Saade G et al. (2002): Transcranial Doppler measurement of cerebral velocity indices as a predictor of preeclampsia. American Journal of Obstetrics and Gynecology, 187 (6): 16671672.

26. Sotiriadis A, Hernandez Andrade E, da Silva Costa F et al. (2019): ISUOG Practice Guidelines: role of ultrasound in screening for and follow up of pre-eclampsia. Ultrasound Obstet Gynecol., 53: 7-22.

27. Lakshmy S, Ziyaulla T, Rose N (2020): The need for implementation of first trimester screening for preeclampsia and fetal growth restriction in low resource settings. The Journal of Maternal-Fetal \& Neonatal Medicine, 20: 1-8.

28. Carbone I, Conforti A, Picarelli S et al. (2020): Circulating nucleic acids in maternal plasma and serum in pregnancy complications: are they really useful in clinical practice? A systematic review. Molecular Diagnosis \& Therapy, 24 (4): 409-431.

29. Wright D, Tan M, O'Gorman N et al. (2019): Predictive performance of the competing risk model in screening for preeclampsia. American Journal of Obstetrics and Gynecology, 220 (2): 199-1.

30. Aksornphusitaphong A, Phupong V (2018): Combination of serum histidine-rich glycoprotein and uterine artery Doppler to predict preeclampsia. Hypertension Research, 41 (4): 275-281.

31. Luewan S, Teja-Intr M, Sirichotiyakul S et al. (2018): Low maternal serum pregnancy-associated plasma proteinA as a risk factor of preeclampsia. Singapore Medical Journal, 59 (1): 55-59.

32. Antsaklis P, Zacharias Fasoulakis M, Diakosavvas M et al. (2019): Association of low maternal pregnancyassociated plasma protein a with adverse perinatal outcome. Cureus, 11 (6): 4912-17. 